\title{
CALCULATION OF TRANSMISSION LINE TRANSIENTS USING POLAR DECOMPOSITION
}

\author{
Bjørn Gustavsen* (M) Adam Semlyen (LF) \\ Department of Electrical and Computer Engineering \\ University of Toronto \\ Toronto, Ontario, Canada M5S 3G4
}

* On leave from the Norwegian Electric Power Research Institute (EFI), Trondheim, Norway.

\begin{abstract}
The paper presents a new transmission line model suitable for the calculation of electromagnetic transients on overhead lines and underground cables. This is achieved by expanding the fitted modes of a conventional line model with constant transformation matrices into simple fractions on the diagonal, and by extending the transformation matrices into rectangular matrices. The new transformation matrices are then refined by a simple iteration procedure based on least squares fitting in the phase domain. The method is demonstrated to give accurate simulation results for both overhead lines and underground cables.
\end{abstract}

\section{INTRODUCTION}

The currently used transmission line models in the EMTP utilize modal decomposition assuming a constant transformation matrix $T$ (CTM-model) calculated at a user-specified frequency. The advantage of this approach is high computational speed as there are only $4 n$ convolutions (for the $n$ modes) to be calculated in the time domain, and because the modes can be fitted accurately with rational functions [1], which makes possible a recursive procedure for the convolutions [2]. An additional advantage of the modal approach is its ability to handle modes with widely different time delays.

It is a well known fact that the transformation matrix $T$ is strongly frequency dependent in the case of cable systems and some overhead lines with asymmetrically arranged conductors. This frequency dependence can in the case of cable systems be taken into account by fitting also the frequency variation of $T$ [3]. This comes at the price of $4 n^{2}$ additional convolutions, although the computational effort can be reduced by a factor of about 3 by using columnwise realization for $T$ [4].

Another way of handling a frequency dependent $T$ is by fitting the transmission line transfer matrices directly in the phase domain [5-7]. The number of convolutions to be calculated is now $4 n^{2}$, but the computational efficiency can be increased by a factor of 3 by using columnwise realization as in [7]. However, it remains a problem to handle modes with widely different time delays, unless these are directly introduced in the fitting process [6].

In this paper we recognize that the conventional CTM-model will normally produce good results in the case of overhead lines. We therefore use the CTM-model to initialize a different model, which is subjected to an iterative refinement. This is done by writing the partial fractions of the fitted modes separately on the diagonal and expanding the transformation matrices into rectangular matrices. The new transformation matrices, $B$ and $C$, are then improved on by a simple iterative procedure, termed "BC-

PE-755-PWRD-0-04-1997 A paper recommended and approved by the IEEE Transmission and Distribution Committee of the IEEE Power Engineering Society for publication in the IEEE Transactions on Power Delivery. Manuscript submitted December 11, 1996; made available for printing April 17, 1997. iteration" in the paper. The expansion of the modes into simple fractions (polar decomposition) gives the additional flexibility needed for taking into account the frequency variation in $T$. This methodology is used separately for the matrix transfer functions of propagation $H$ and characteristic admittance $Y_{c}$, where the modes are fitted using the method by optimal scaling developed in [4]. The total number of partial fractions (poles) is in the new model equal to that of the CTM-model used for initialization, but the transformation matrices are now larger. In the paper we also show that sufficiently accurate results can be achieved by putting the partial fractions as pairs on the diagonal, which has the advantage of reducing the size of the transformation matrices in half.

A noteworthy advantage of the new method over the proposed phase domain models in [5] and [7] is its ability of handling modes with widely different time delays. This is because a separate time delay can be assigned to each diagonal element.

Calculated results in the paper demonstrate that the model gives accurate results for both overhead lines and underground cables.

\section{METHODOLOGY}

\subsection{Traveling wave equations}

The transmission line model in the paper is based on the traveling wave approach. At each end of the transmission line the traveling wave solution can be formulated in the frequency domain as

$$
Y_{c} v-i=2 i_{i}=2 H i_{\text {far }}
$$

where $v$ and $i$ are the line to ground voltages and line currents, respectively. $i_{i}$ is the incident current wave and $i_{f a r}$ is the reflected current wave from the opposite line end. The wave propagation is characterized by the propagation function $H$ and the characteristic admittance $Y_{c}$, defined by:

$$
\begin{gathered}
H=\exp (-\sqrt{Y Z} l) \\
Y_{c}=Z^{-1} \sqrt{Z Y}
\end{gathered}
$$

$Z$ and $Y$ are the series impedance and shunt admittance per unit length of the transmission line which for an $n$-conductor system have dimension $n \times n$.

\subsection{Modal decomposition}

$H$ and $Y_{c}$ are diagonalized into $H^{m}$ and $Y_{c}^{m}$ using a real, constant transformation matrix $T_{I 0} \approx T_{I}$ :

$$
\begin{aligned}
& H \approx T_{I 0} H^{m} T_{I 0}^{-1} \\
& Y_{c} \approx T_{I 0} Y_{c}^{m} T_{I 0}^{T}
\end{aligned}
$$

where $T_{I}$ is the right eigenvector matrix for $Y Z$. Each mode $i$ of $H^{m}$ and $Y_{c}^{m}$ is approximated by a sum of partial fractions using the method of vector fitting introduced in [4] :

$$
H_{i}^{m} \approx \sum_{k=1}^{N_{i}} \frac{r_{i k}}{s-p_{i k}} e^{-s \tau_{i}}
$$




$$
Y_{c i}^{m} \approx d_{i}+\sum_{k=1}^{N_{i}} \frac{r_{i k}}{s-p_{i k}}
$$

$\left(r_{i k}\right.$ and $p_{i k}$ are not the same in (6) and (7).)

\subsection{Polar decomposition}

The modal domain model of (4) - (7) will now be expanded into a polar model in which each partial fraction in (6) and (7) appears separately on the diagonal. (In order to simplify the notation we write $T$ instead of $T_{I 0}$ and $S$ instead of $T_{I 0}^{-1}$ ).

Let us first consider the fitting of $H$. For instance, in the case of a two-conductor system we get from (4) and (6):

$$
H \approx T\left[\begin{array}{cc}
\sum_{k} \frac{r_{1 k} e^{-j \omega \tau_{1}}}{s-p_{1 k}} & 0 \\
0 & \sum_{k} \frac{r_{2 k} e^{-j \omega \tau_{2}}}{s-p_{2 k}}
\end{array}\right] S
$$

We note that each mode multiplies a column $t$ in $T$ and a row $s$ in $S$. The modes in (8) can therefore be expanded into simple fractions on the diagonal by repeating columns in $T$ and rows in $S$. (A similar procedure was also used in [8].) Assuming that each mode has been fitted with 2 poles we get:

$H \approx\left[\begin{array}{llll}t_{1} & t_{1} & t_{2} & t_{2}\end{array}\right]\left[\begin{array}{cccc}\frac{r_{11} e^{-j \omega \tau_{1}}}{s-p_{11}} & 0 & 0 & 0 \\ 0 & \frac{r_{12} e^{-j \omega \tau_{1}}}{s-p_{12}} & 0 & 0 \\ 0 & 0 & \frac{r_{21} e^{-j \omega \tau_{2}}}{s-p_{21}} & 0 \\ 0 & 0 & 0 & \frac{r_{22} e^{-j \omega \tau_{2}}}{s-p_{22}}\end{array}\right]\left[\begin{array}{l}s_{1} \\ s_{1} \\ s_{2} \\ s_{2}\end{array}\right](9)$

which in a shorter form can be written as :

$$
H \approx C_{H} G_{H}(s) B_{H}
$$

It should be noted that (8) and (10) give identical approximations for $H$, but $C_{H}$ and $B_{H}$ are larger than $T$ and $S$.

In the case of the characteristic admittance, we similarly get

$$
Y_{c} \approx C_{Y_{c}} G_{Y_{c}}(s) B_{Y_{c}}+D
$$

where $C_{Y_{c}}$ and $B_{Y_{c}}$ are calculated by repeating columns from $T_{I 0}$ and rows from $T_{I 0}^{T}$, respectively. $G_{Y_{c}}$ is given by the partial fractions in (7), but without the scalars $d_{i}^{c} . D$ in (11) is specified to be equal to $\operatorname{Re}\left\{Y_{c}\right\}$ at the highest frequency point used in the fitting of $Y_{c}^{m}$.

\subsection{BC-iterations}

The matrices $C$ and $B$ in (10) and (11) are now refined by a simple iterative procedure. For $H$, e.g., this is done as follows :

1. With $C$ known, calculate $B$ as the least squares solution of the linear equation

$$
[C G(s)] B=H(s)
$$

2. With the improved $B$, calculate $C$ as the least squares solution of the linear equation

$$
\left[B^{T} G(s)\right] C^{T}=H^{T}(s)
$$

Steps 1 and 2 are repeated until satisfactory results have been achieved. It should be noted that (12) and (13) are split into their real and imaginary parts to ensure realness for the resulting $C$ and $B$. (The procedure for $Y_{c}$ is similar and will therefore not be repeated here.)

Because each of the steps 1 and 2 applies a least squares fit to $H$ (or $Y_{c}$ ) directly, the iterations will never diverge. The convergence of the $\mathrm{BC}$-iterations is remarkably fast, both for $H$ and $Y_{c}$. Typically, the iterations can be terminated after only 3-5 steps. In addition, the actual solving of equations (12) and (13) is fast because $G(s)$ is calculated only a single time, and because the size of the left matrix in (12) and (13) is moderate. If, for instance, the modes of a six-conductor line are each fitted with 10 poles and the equation is written for 50 frequency points we find that the left matrix has dimension $(600 \times 60)$. This result is contrary to Gauss-Newton iterations [8] which converge slowly and have a very large Jacobian.

When the modes of $H$ are fitted using the method of optimal scaling [4] used in the paper, some of the poles may appear in complex conjugate pairs. The handling of such pairs is described in the Appendix.

\subsection{Time domain implementation}

Inserting the polar approximations for $H(10)$ and $Y_{c}(11)$ into the traveling wave solution (1) gives

$$
C_{Y_{c}} G_{Y_{c}} B_{Y_{c}} v-i=2 C_{H} G_{H} B_{H} i_{f a r}
$$

Equation (14) is transformed into the time domain using convolutions :

$$
C_{Y c}\left(G_{Y c} * v^{p}\right)-i=2 C_{H}\left(G_{H}^{*} i_{f a r}^{p}\right)
$$

where

$$
v^{p}=B_{Y_{c}} v ; i_{\text {far }}^{p}=B_{H} i_{f a r}
$$

Note that in (15) and (16), all quantities are in the time domain The line model described by (15) and (16) is converted into a Norton equivalent consisting of a constant conductance matrix in parallel with a current source of past history values, assuming trapezoidal integration.

It should be noted that the traveling waves in (14) are in the phase domain whereas the traveling waves in the CTM-line are in the modal domain. This is because the new model does not satisfy the relationship $T_{V}=T_{I}^{-T}$, where $T_{V}$ is the right eigenvector matrix of $Z Y$. The consequence of this is that while the CTM-line needs for each line end only two transformations $\left(T_{I}\right.$ and $\left.T_{I}^{T}\right)$, the polar model needs four transformations $\left(C_{Y_{c}}, B_{Y_{c}}, C_{H}, B_{H}\right)$.

\section{CALCULATED RESULTS FOR OVERHEAD LINE}

\subsection{Transmission line data}

Figure 3 shows a system of two single circuit lines $(230 \mathrm{kV}$ and $115 \mathrm{kV}$ ) sharing the same right-of-way. In the following it is assumed that the line length is $100 \mathrm{~km}$, and that the phase conductors are untransposed.

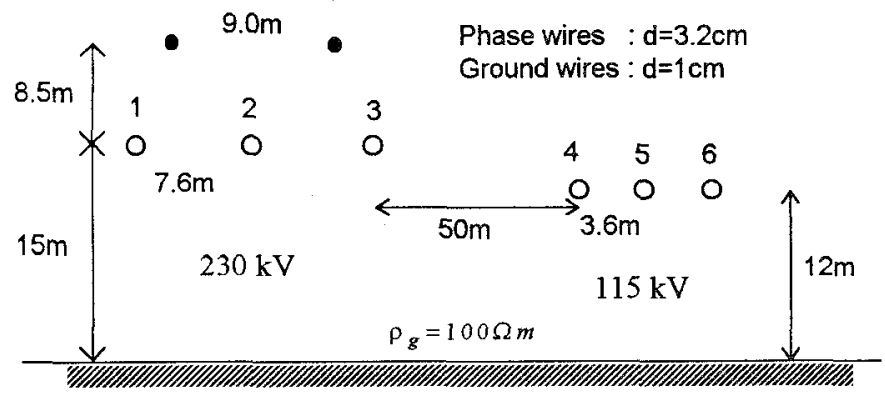

Fig. 3 Two single circuit overhead lines on the same right-of-way

\subsection{Modal decomposition}

The propagation $H$ and characteristic admittance $Y_{c}$ were diagonalized using a real, constant transformation matrix calculated at $1 \mathrm{MHz}$. The modes were fitted in the range $1 \mathrm{~Hz}-$ $1 \mathrm{MHz}$ using six and five poles, respectively. Figures 4 and 5 show the fitted modes for $H$ and $Y_{c}$, calculated by the method of 
optimal scaling [4]. Also is shown the magnitude of the complex deviation. It is seen that the modes have been fitted very accurately-the fitted curves are superimposed on the accurate ones.



Fig. 4 Fitted elements of $H^{m}$ (sixth order approximation)

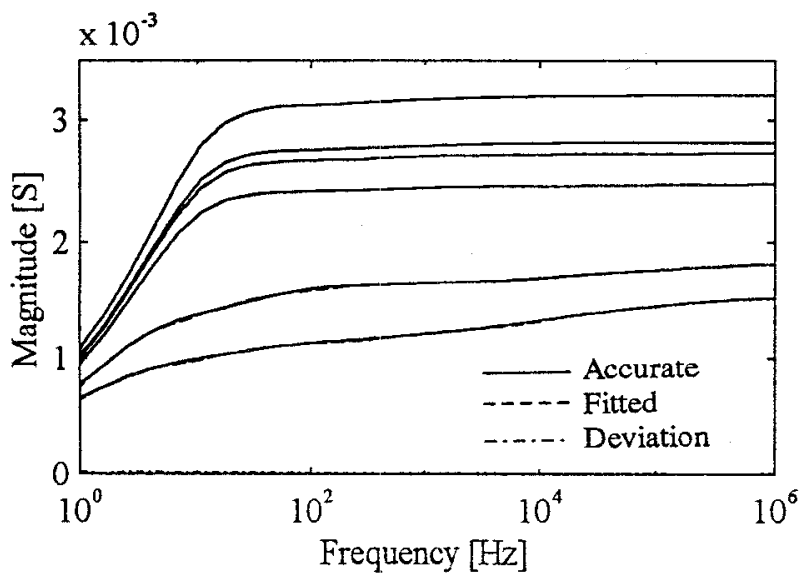

Fig. 5 Fitted elements of $Y_{c}^{m}$ (fifth order approximation)

\subsection{Polar decomposition for $H$}

Using the constant transformation matrix and the fitted modes, the matrices $B, C$ and $G$ in (10) were initialized. Figure (6) shows the first column of $H$ for the initialized model ( 0 iterations), which corresponds in accuracy to the CTM-line. It is seen that a significant error is present for all elements.

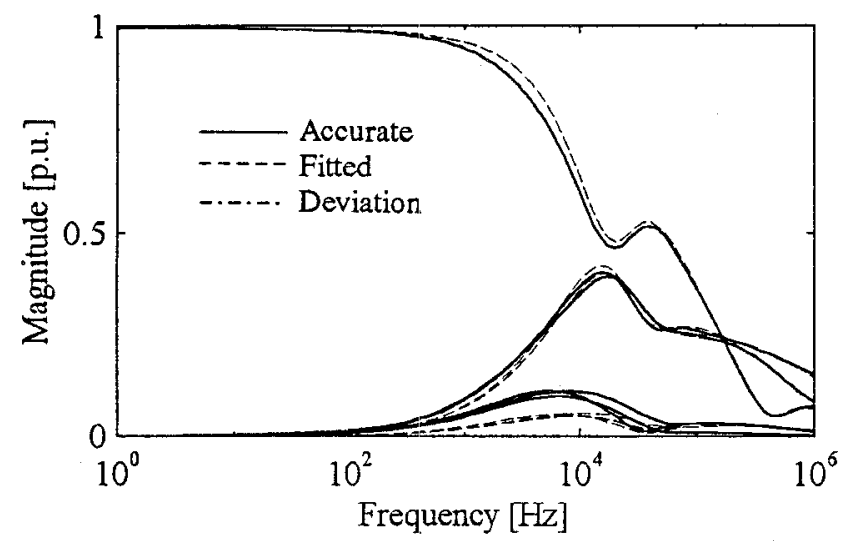

Fig. 6 First column of $\mathrm{H}$ by initialized model ( 0 iterations)

Figure 7 shows the same results after three BC-iterations. It is seen that the accuracy is now much higher than in figure 6 . The iterations resulted in that the root-mean-square error (RMS-error) for $H$ (all columns) decreased from $21.2 \mathrm{E}-3$ to $2.2 \mathrm{E}-3$, which is an improvement by a factor of 10 .

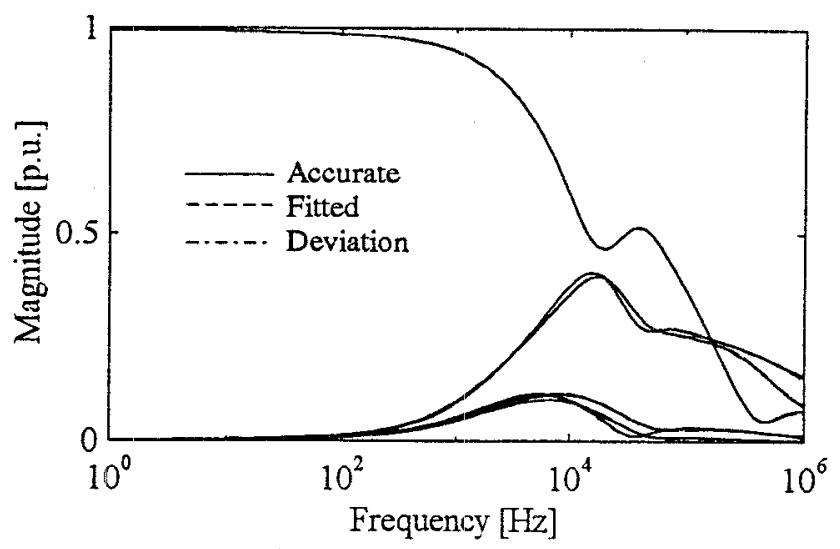

Fig. 7 First column of $H$ after 3 BC-iterations

The accuracy of the polar decomposition is of course dependent on how many poles are used in the fitting of the modes. Table 1 shows how the RMS-error of the fit of $H$ changes with the number of poles used in the fitting (all modes are fitted with the same number of poles). Also is shown how the error decreases with the number of $\mathrm{BC}$-iterations.

Table 1 Root-Mean-Square error of $H(\times 1 E-3)$ Order of fitting $(\mathrm{N})$

$\begin{array}{lccccccc}\text { iter } & \mathrm{N}=3 & \mathrm{~N}=4 & \mathrm{~N}=5 & \mathrm{~N}=6 & \mathrm{~N}=7 & \mathrm{~N}=8 & \mathrm{~N}=15 \\ 0 & 24.2 & 22.0 & 21.3 & 21.2 & 21.2 & 21.2 & 21.1 \\ 1 & 12.7 & 7.6 & 3.5 & 2.6 & 2.0 & 1.9 & 0.27 \\ 2 & 12.2 & 7.1 & 3.3 & 2.4 & 1.9 & 1.6 & 0.15 \\ 3 & 12.2 & 7.0 & 3.2 & 2.2 & 1.8 & 1.5 & 0.12 \\ 10 & 12.1 & 6.9 & 3.1 & 2.0 & 1.6 & 1.2 & 0.09\end{array}$

From table 1 we see the following :

- The accuracy of the initialized model (iteration 0 ) does not improve by using a large number of poles. This is of course due to the frequency dependency of the transformation matrix.

- The BC-iterations converge very fast-there is not much improvement from the third to the tenth iteration.

- The improvement in accuracy achieved by iteration increases strongly as the number of poles of the fit increases. The improvement is by a factor 2 when $\mathrm{N}=3$ and a factor 234 when $\mathrm{N}=15$.

- Six poles gives an RMS-error of $0.2 \%$, which is probably sufficiently accurate for most practical studies.

Table 2 shows the accuracy of the fitted modes $\left(H^{m}\right)$. By comparing table 2 with the last row in table 1 it is seen that $H$ is fitted more accurately than the original modes.

Table 2 Root-Mean-Square error of $H^{m}(\times 1 E-3)$ Order of fitting $(\mathrm{N})$

$\begin{array}{ccccccc}\mathrm{N}=3 & \mathrm{~N}=4 & \mathrm{~N}=5 & \mathrm{~N}=6 & \mathrm{~N}=7 & \mathrm{~N}=8 & \mathrm{~N}=15 \\ 39.5 & 18.0 & 9.2 & 6.0 & 5.1 & 4.3 & 0.3\end{array}$




\subsection{Polar decomposition for $Y_{0}$}

The $B, C$ and $G$-matrices in (11) were initialized using the constant transformation matrix and the fitted modes previously shown in figure 5 (fifth order fit).

Figure 8 shows the first column of $Y_{c}$ after initialization (0 iterations), which corresponds to the accuracy of the CTM-line. It is seen that a significant error occurs at frequencies below $10 \mathrm{kHz}$.

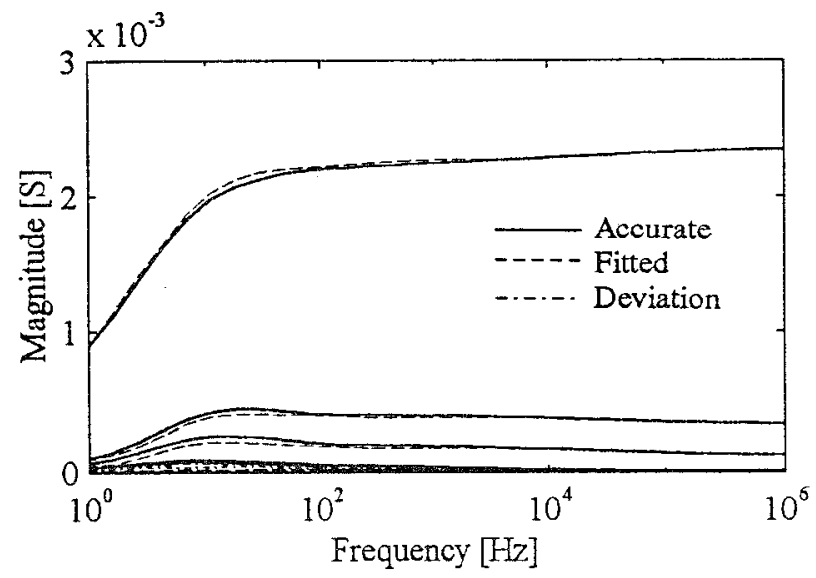

Fig. 8 First column of $Y_{c}$ by initialized model (0 iterations)

Figure 9 shows the accuracy of the fit after 3 BC-iterations. It is seen that the fit is now quite accurate.

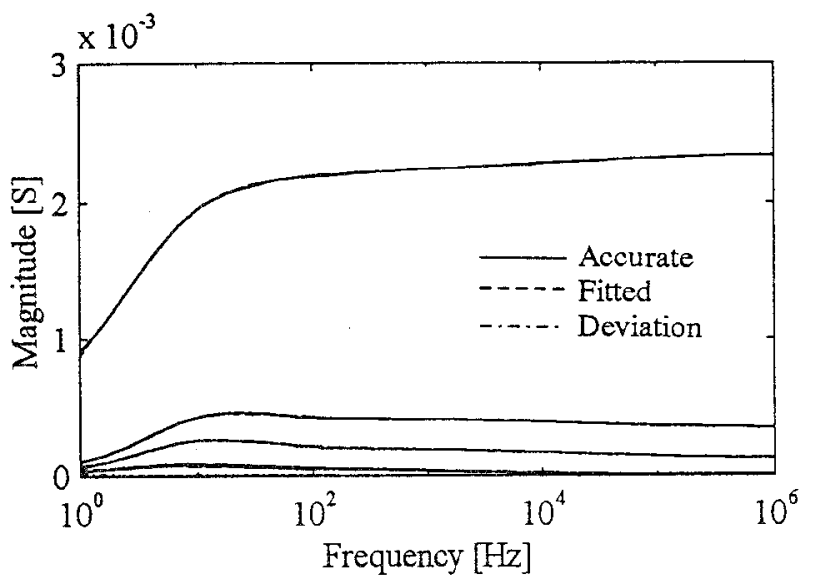

Fig. 9 First column of $Y_{c}$ after 3 iterations

Table 3 shows how the accuracy of the resulting fit depends on the number of BC-iterations and the number of poles used for the fitting of the modes $\left(Y_{c}^{m}\right)$. (All modes are fitted with the same number of poles, N.)

Table 3 Root-Mean-Square error of $Y_{c}(\times 1 E-6)$ Order of fitting $(\mathrm{N})$

$\begin{array}{lccccccc}\text { iter } & \mathrm{N}=3 & \mathrm{~N}=4 & \mathrm{~N}=5 & \mathrm{~N}=6 & \mathrm{~N}=7 & \mathrm{~N}=8 & \mathrm{~N}=15 \\ 0 & 35.6 & 28.6 & 28.2 & 28.0 & 28.0 & 27.9 & 27.9 \\ 1 & 22.6 & 7.5 & 6.7 & 3.8 & 2.2 & 1.3 & 0.22 \\ 2 & 22.2 & 7.4 & 6.6 & 3.7 & 2.0 & 1.3 & 0.22 \\ 3 & 21.6 & 7.3 & 6.4 & 3.6 & 1.9 & 1.2 & 0.21 \\ 10 & 21.2 & 7.0 & 5.9 & 3.3 & 1.9 & 1.1 & 0.21\end{array}$

The results in table 3 resemble those for the propagation in table 1 : Convergence is very fast and the improvement of the fit achieved by BC-iterations increases with the number of poles used in the fitting. The improvement is seen to vary from a factor of 1.6 when $\mathrm{N}=3$ to a factor of 141 when $\mathrm{N}=15$.

Table 4 below shows that the modes became fitted very accurately as the number of poles was increased. Thus, the relatively poor fit for $Y_{c}$ achieved with 0 iterations in table 3 is due to the frequency variation of the transformation matrix.

Table 4 Root-Mean-Square error of $Y_{c}^{m}(\times 1 E-6)$ Order of fitting $(\mathrm{N})$

$\begin{array}{ccccccc}\mathrm{N}=3 & \mathrm{~N}=4 & \mathrm{~N}=5 & \mathrm{~N}=6 & \mathrm{~N}=7 & \mathrm{~N}=8 & \mathrm{~N}=15 \\ 85.8 & 24.9 & 15.3 & 9.7 & 7.5 & 3.6 & 0.82\end{array}$

\subsection{Time domain simulation}

In the following we show a simulation example for the line in figure 3. The modes of $H$ and $Y_{c}$ were fitted with 6 and 5 poles, respectively, and $H$ and $Y_{c}$ were fitted using polar decomposition with $10 \mathrm{BC}$-iterations.

In order to assess the accuracy of the result we also calculate an alternative solution by the full phase domain model in [7]. The columns of $H$ and $Y_{c}$ were fitted using 20 and 10 poles, respectively.

In the simulation we energize the $230 \mathrm{kV}$ line with symmetric voltages and disconnect the line after $20 \mathrm{~ms}$. This is shown in figure 10.

close : $t=0$ open : $\mathrm{t}=20 \mathrm{~ms}$

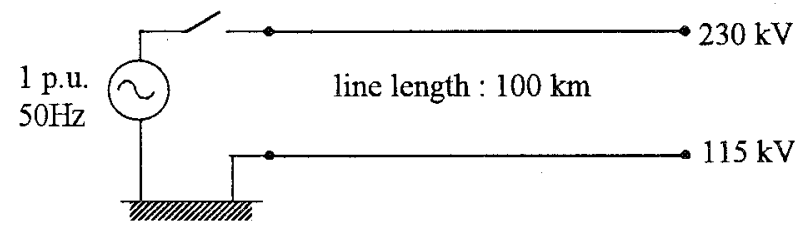

Fig. 10 Symmetric energization of $230 \mathrm{kV}$ line followed by disconnection

Figure 11 shows the resulting voltages at the receiving end for the two outer phases of the $230 \mathrm{kV}$ line. (The numbering of conductors is given in figure 3 ). It is seen that the simulated voltages by the method of polar decomposition are in close agreement by the result from the phase domain simulation. Also, both methods simulate the trapped voltage without sign of drifting.

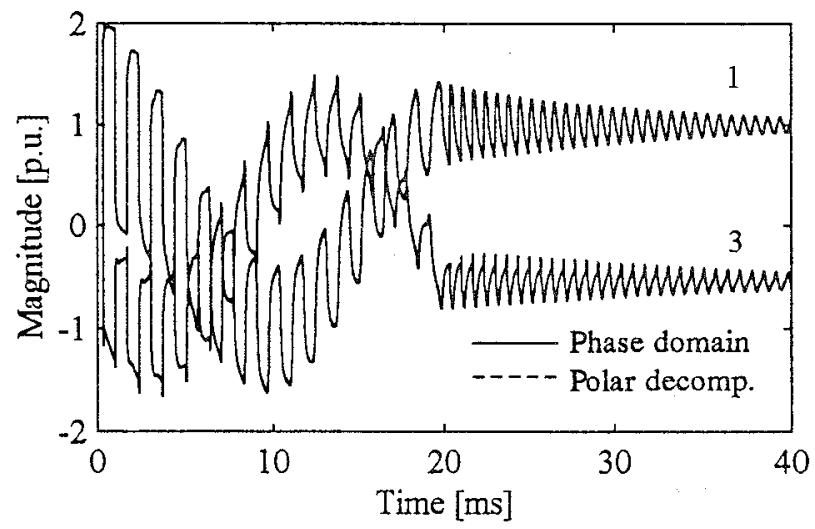

Fig. 11 Simulated voltage on outer phases of $230 \mathrm{kV}$ line.

Figure 12 shows the induced voltage on the mid-phase (figure 3) of the $115 \mathrm{kV}$ line, as calculated by the polar model and the phase domain model in [7]. In addition is shown the voltage calculated by the polar model without BC-iterations. It is seen that 
the $\mathrm{BC}$-iterations give a big reduction of the error in the simulation .

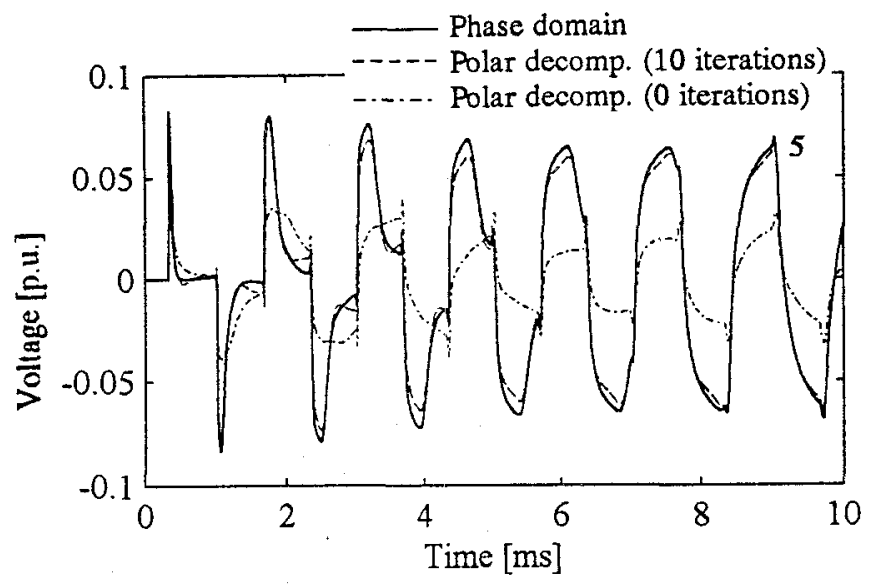

Fig. 12 Simulated voltage of mid-phase of $115 \mathrm{kV}$ line.

\section{CALCULATED RESULTS FOR UNDERGROUND CABLE}

In the following we show results for the $66 \mathrm{kV}$ cable system below. The cable length is assumed to be $5 \mathrm{~km}$ in all calculations. The cable data have previously been shown in [4] and will therefore not be repeated here.

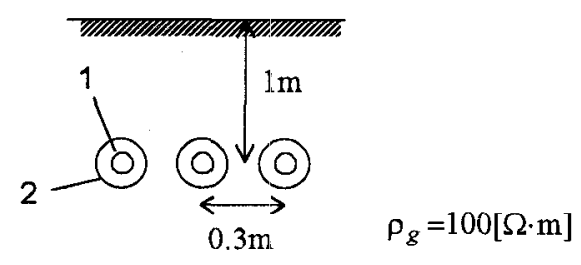

Fig. 133 single core coaxial cables.

$H$ and $Y_{c}$ were diagonalized using a real, constant transformation matrix calculated at $1 \mathrm{MHz}$, and the modes were fitted using 7 and 5 poles, respectively. The fit was found to be quite accurate. Using the transformation matrix and the fitted modes, the polar modal model was initialized.

Figures 14 and 15 show the fitted elements in the second column of $H$, after 0 and $3 \mathrm{BC}$-iterations, respectively. (The conductor numbering is given in figure 13). It is seen that the accuracy is fairly good even without any iterations. After $3 \mathrm{BC}$ iterations the RMS-error for $H$ was reduced from $8.4 \mathrm{E}-3$ to $3.0 \mathrm{E}-3$.



Fig. 14 First column of $H$ by initialized model ( 0 iterations).

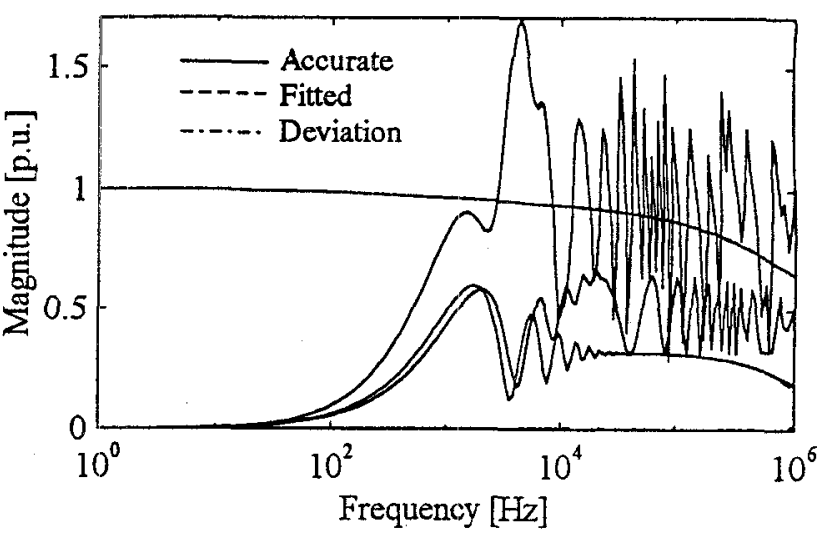

Fig. 15 First column of $H$ after 3 BC-iterations.

Figures 16 and 17 show the fitted elements in the second column of $Y_{c}$, after 0 and $3 \mathrm{BC}$-iterations, respectively. It is seen that the error in the first case is very large, but becomes negligible after the BC-iterations. This made the RMS-error in the fit for $Y_{c}$ decrease from $45.5 \mathrm{E}-4$ to $1.6 \mathrm{E}-4$, which is by a factor of 27 .

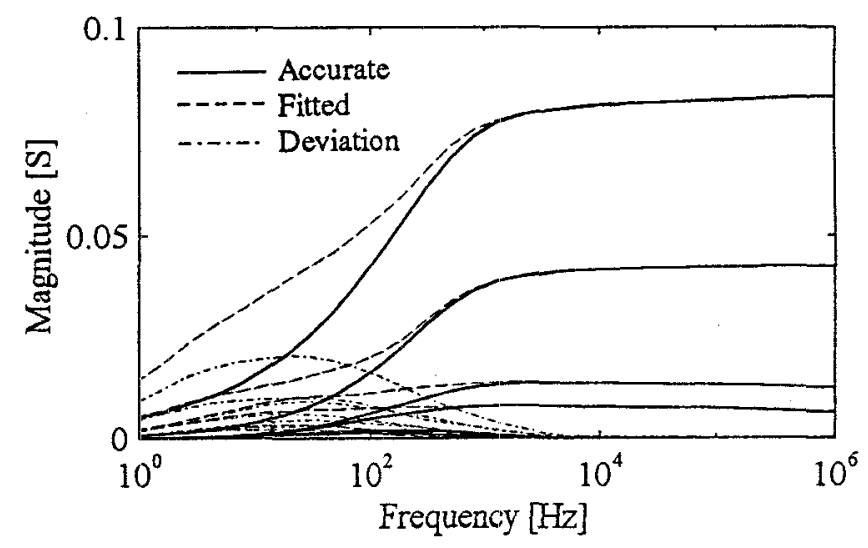

Fig. 16 Second column of $Y_{c}$ by initialized model ( 0 iterations).

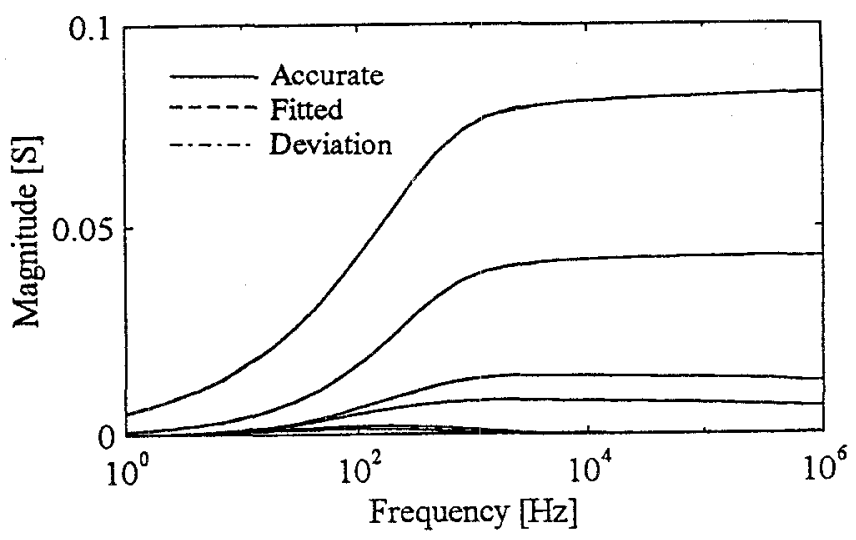

Fig. 17 Second column of $Y_{c}$ after 3 BC-iterations.

In the following we show a time domain simulation for the resulting cable system ( $10 \mathrm{BC}$-iterations were used for $H$ and $Y_{c}$ ). A 1 .p.u. step voltage was applied to the core of the leftmost cable (figure 18), with all other cores and the sheaths grounded at this end. At the other end (receiving end) all cores and sheaths were grounded. 


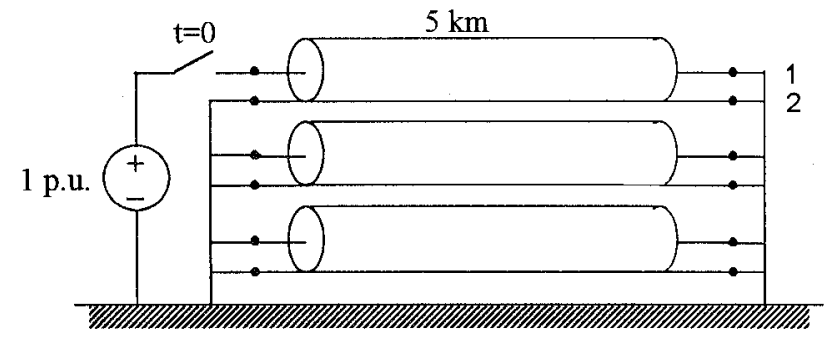

Fig. 18 Short circuit test

Figure 19 shows the calculated current flowing in the sheath of the energized cable, at the sending end. In addition we have calculated the current by the method of frequency dependent transformation matrices [3], using the implementation in [4]. It is seen that a good agreement between the simulated responses is achieved.

In the figure we have also shown the calculated result by the polar model when no $\mathrm{BC}$-iterations were used, corresponding to figures 14 and 16. It is seen that a very large deviation now occurs. This result is mainly due to the large error in $Y_{c}$ (figure 16), which was caused by neglecting the frequency dependence of the transformation matrix.

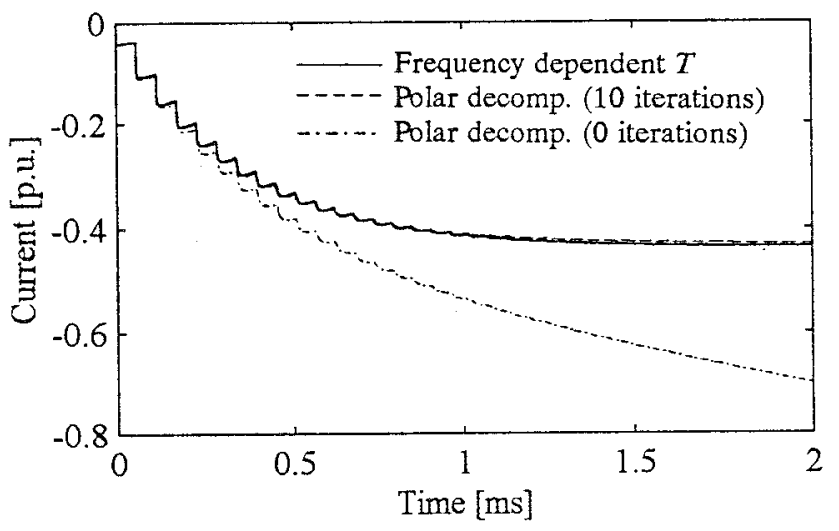

Fig. 19 Induced sheath current in energized cable

\section{PARTIAL POLAR DECOMPOSITION}

In the method of polar decomposition each partial fraction in the fitted modes is written separately on the diagonal of the $G$ matrix in (10) and (11). Thus, if there are $n$ conductors and each mode is fitted with $N$ poles, then $C$ and $B$ each have $N n^{2}$ elements, as opposed to $n^{2}$ elements for the CTM-line. In the case of systems with many conductors (multi-circuit lines), $C$ and $B$ may therefore result in a substantial computational effort in the time domain simulations.

The size of $C$ and $B$ can easily be reduced by making a partial decomposition of the fitted modes, but comes at the price of reduced accuracy of the resulting fitting for $H$ and $Y_{c}$. A practical compromise is to place the partial fractions in pairs on the diagonal of $G$ in (10) and (11), which results in the size of $C$ and $B$ being reduced by a factor of 2 . The pairs are selected in the following way: For each mode, sort the fractions so that the poles come in increasing order. Consecutive fractions are then taken as pairs. (The columns of $C$ and the rows of $B$ are sorted accordingly to get the correct initialization.) The BC-iterations are carried out as previously described in section 2.4 .

Table 5 shows the accuracy of the fitted $H$-matrix for the sixconductor overhead line in figure 3 , for different number of poles used in the fitting of each mode, after $10 \mathrm{BC}$-iterations. Similar results is shown in Table 6 for $Y_{c}$. Comparing the results with those previously obtained with full polar decomposition (tables 1 and 3) shows that the fit for a given number of poles is of the order of 2-3 times less accurate.

Table 5 Root-Mean-Square error of $H(\times 1 E-3)$. Partial fractions in pairs on diagonal.

Order of fitting $(\mathrm{N})$

$\begin{array}{lcccc}\text { iter } & \mathrm{N}=4 & \mathrm{~N}=6 & \mathrm{~N}=8 & \mathrm{~N}=10 \\ 0 & 22.0 & 21.2 & 21.2 & 21.1 \\ 10 & 11.6 & 6.31 & 4.18 & 3.57\end{array}$

Table 6 Root-Mean-Square error of $Y_{c}(\times 1 E-6)$. Partial fractions in pairs on diagonal.

Order of fitting $(\mathrm{N})$

$\begin{array}{lllll}\text { iter } & \mathrm{N}=4 & \mathrm{~N}=6 & \mathrm{~N}=8 & \mathrm{~N}=10 \\ 0 & 28.6 & 28.0 & 27.9 & 27.9 \\ 10 & 16.1 & 4.79 & 3.06 & 3.34\end{array}$

Further improvement in accuracy was achieved by including the residuals of each pair in the iterations. This was done by introducing an additional stage after solving for $C$ and $B$. However, it was found that the increase in accuracy was only marginal.

\section{DisCUSSION}

In this paper we have shown that a transmission line model based on fitted modes and a constant, square transformation matrix (CTM-model) can be expanded into a new decomposition with simple fractions on a diagonal matrix $(G)$ and rectangular transformation matrices $(C$ and $B)$. This expanded polar decomposition (PD-model) has the same accuracy as the original CTM-model, but is then improved on by an iterative procedure in which the $C$ and $B$ matrices are modified (BC-iteration).

In the following we argue that the PD-model is not very dependent on the accuracy of the CTM-model used for initialization. The transformation matrix used for initialization of $C$ and $B$ may depend strongly on the frequency point selected for diagonalization. However this is not a problem as we have found that even a quite strong perturbation for the starting values of $B$ and $C$ has only a small effect on the outcome of the BC-iterations. The selection of the frequency point for diagonalization also influences the shape of the modes and therefore also the fitted modes used in the initialization. Each fitted mode is written separately on the diagonal of $G$ as a residual over a pole : $r /(s-p)$. Because each residual multiplies a column in $C$ and a row in $B$ it follows that inaccurate residuals will be compensated for in the $\mathrm{BC}$-iterations by a scaling of columns in $C$ and rows in $B$. On the other hand, poorly selected poles cannot be compensated for, so good poles are surely important. However, the resulting poles in the CTM-model do not change much by the frequency point selected for diagonalization, and small displacements of the poles will partly be compensated for in the BC-iterations.

In the calculated examples we used a transformation matrix calculated at $1 \mathrm{MHz}$, which is certainly not the best choice for an overhead line. This was done for the purpose of showing that highly accurate results can be achieved without using a very good starting point. Thus, in a setup routine it is possible to use a standard frequency point for diagonalization, thus eliminating the need for user interaction. For illustration, if for the overhead line in figure 3 we used a transformation matrix evaluated at $f_{0}=5 \mathrm{kHz}$ we got the resulting accuracy for propagation $H$ : 
Table 7 Root-Mean-Square error of $H(\times 1 E-3)$ Order of fitting $(\mathrm{N})$

$\begin{array}{lrrrrrrc}\text { iter } & \mathrm{N}=3 & \mathrm{~N}=4 & \mathrm{~N}=5 & \mathrm{~N}=6 & \mathrm{~N}=7 & \mathrm{~N}=8 & \mathrm{~N}=15 \\ 0 & 38.9 & 14.0 & 8.8 & 8.6 & 8.5 & 8.5 & 8.5 \\ 10 & 12.5 & 5.1 & 2.9 & 1.8 & 1.5 & 0.88 & 0.07\end{array}$

Comparison with the corresponding results in table $1\left(f_{0}=1 \mathrm{MHz}\right)$ shows that not much improvement was achieved after $10 \mathrm{BC}$ iterations, although the initialized model ( 0 iterations) is improved in accuracy by a factor of about 2.5 .

In section 5 we placed the partial fractions of the modes in pairs on the diagonal of $G$. This had the advantage of reducing the size of $C$ and $B$, which in the case of lines with many conductors would lead to computational savings in the time step loop. For the six-conductor line examined in this paper this method resulted in a substantial reduction in accuracy as compared to the full polar decomposition, although the improvement over the initialized model ( 0 iterations) was still large.

It should be noted that there is a significant difference between the method of polar decomposition and our previous approach of columnwise fitting directly in the phase domain [7]. With the polar approach, the poles are calculated from the modes, and the poles from all modes are used in the fitting of all phase domain columns via the $B$ and $C$ matrices. In the phase domain approach, each column was fitted independently of the others, and each column was given a separate realization. An important improvement of the new model is its ability to handle modes with widely different time delays, as a separate time delay can be assigned to each partial fraction. This makes the new model very useful for the simulation of cable systems where the modal time delays can be very different.

\section{CONCLUSIONS}

In this paper we have developed a new transmission line model suitable for the calculation of electromagnetic transients in both overhead lines and underground cables.

The new model is based on fitting the modes for propagation, $H$, and characteristic admittance, $Y_{c}$, assuming a constant transformation matrix, as in the currently used line models in the EMTP. The new feature is that the partial fractions of each mode are written separately on the diagonal matrix and that the transformation matrices are extended into rectangular matrices. The new transformation matrices are then improved by a simple iterative procedure based on least squares fitting in the phase domain. The method is applied separately to the transfer matrices for propagation and characteristic admittance.

Calculated results in the paper have shown that the new method gives highly accurate results for both overhead lines and underground cables. The success achieved with this model is due to that the fact that the extension of the transformation matrices gives sufficient flexibility to account for a frequency dependent transformation matrix, while at the same time individual time delays can be assigned to the partial fractions on the diagonal matrix.

\section{ACKNOWLEDGEMENTS}

Financial assistance by the Natural Sciences and Engineering Research Council of Canada is gratefully acknowledged. The first author wishes to express his gratitude to the Norwegian Electric Power Research Institute (EFI), Trondheim, Norway, for granting and financing his leave at the University of Toronto.

\section{REFERENCES}

[1] J. R. Marti, "Accurate Modelling of Frequency-Dependent Transmission Lines in Electromagnetic Transient Simulations", IEEE Trans. PAS, vol. 101, no. 1, January 1982, pp. 147-157.

[2] A. Semlyen and A. Dabuleanu, "Fast and Accurate Switching Transient Calculations on Transmission Lines With Ground Return Using Recursive Convolutions", IEEE Trans. PAS, vol. 94, March/April 1975, pp. 561-571.

[3] L. Marti, "Simulation of Transients in Underground Cables With Frequency-Dependent Transformation Matrices", IEEE Trans. PWRD, vol. 3, no. 3, July 1988, pp. 1099-1110.

[4] B. Gustavsen and A Semlyen, "Simulation of Transmission Line Transients Using Vector Fitting and Modal Decomposition", accepted for publication in the IEEE Transactions and presentation at the IEEE PES Winter Meeting, New York, 1997.

[5] H. V. Nguyen, H. W. Dommel, and J. R. Marti, "Direct Phase-Domain Modelling of Frequency-Dependent Overhead Transmission Lines", paper no. 96 SM 458-0-PWRD.

[6] T. Noda, N. Nagaoka and A. Ametani, "Phase Domain Modeling of Frequency-Dependent Transmission Lines by Means of an ARMA Model", IEEE Trans. PWRD, vol. 11, no. 1, January 1996, pp. 401-411.

[7] B. Gustavsen and A. Semlyen, "Combined Phase and Modal domain Calculation of Transmission Line Transients Based on Vector Fitting", accepted for publication in the IEEE Transactions and presentation at the IEEE PES Winter Meeting, New York, 1997.

[8] A.O Soysal and A. Semlyen, "State Equation Approximation of Transfer Matrices and Its Application to the Phase Domain Calculation of Electromagnetic Transients", IEEE Trans. PWRD, no. 1, February 1994, pp. $420-428$.

\section{APPENDIX}

\section{Complex conjugate Pairs in BC-iterations}

In the following we show how complex conjugate pairs in the fitted modes of the CTM-model were handled in the initialization of the polar model.

Let

$$
f=\frac{r^{\prime}+j r^{\prime \prime}}{s-\left(p^{\prime}+j p^{\prime \prime}\right)}+\frac{r^{\prime}-j r^{\prime \prime}}{s-\left(p^{\prime}-j p^{\prime \prime}\right)}
$$

be a complex pair with corresponding column $t_{i}$ in the transformation matrix and row $s_{i}$ in the inverse transformation matrix (see 8). In the polar model this pair gives two columns, $C^{\prime}$, in the $C$-matrix, two rows, $B^{\prime}$, in the $B$-matrix and a two-bytwo matrix, $G^{\prime}$, on the diagonal of $G$. These are given as :

$$
\begin{gathered}
C^{\prime}=\left[\begin{array}{ll}
r^{\prime} T_{i} & r^{\prime \prime} T_{i}
\end{array}\right] \\
B^{\prime}=\left[\begin{array}{c}
2 s_{i} \\
0
\end{array}\right] \\
G^{\prime}(s)=\left[\begin{array}{cc}
s-p^{\prime} & -p^{\prime \prime} \\
-p^{\prime \prime} & s-p^{\prime \prime}
\end{array}\right]^{-1}
\end{gathered}
$$

Thus, the resulting $G$-matrix becomes block-diagonal but $B$ and $C$ remain real.

\section{BIOGRAPHIES}

Bjørn Gustavsen was born in 1965 in Harstad, Norway. He received the M.Sc. degree in Electrical Engineering from The Norwegian Institute of Technology, Trondheim, in 1989, and the Dr. Ing. Degree in 1993. Since 1994 he has been working at the Norwegian Electric Power Research Institute, mainly in the field of transient studies. He is currently on leave at the Department of Electrical and Computer Engineering, University of Toronto.

Adam Semlyen was born in 1923 in Rumania where he obtained a Dipl. Ing degree and his Ph.D. He started his career there with an electric power utility and held academic positions at the Polytechnic Institute of Timisoara. In 1969 he joined the University of Toronto where he is a professor in the Department of Electrical and computer engineering, emeritus since 1988. His research interests include steady state and dynamic analysis as well as computation of electromagnetic transients in power systems. 


\section{Discussion}

Taku Noda (Central Research Institute of Electric Power Industry - CRIEPI, Tokyo, Japan) and Akihiro Ametani (Doshisha University, Kyoto, Japan): We would like to congratulate the authors for developing the new line model based on the polar decomposition.

The proposed polar decomposition is mathematically well-structured and efficient to handle modal traveling-time delays in the phase domain. Furthermore, it would be valuable if the physical meaning of the polar decomposition is clarified. From our understanding, the modal theory determines the weighting coefficients of modal components, having different traveling times, to be included in the phasedomain quantities. On the other hand, the polar decomposition determines the weighting coefficients of poles contributing to wave deformation. The comments of the authors are highly appreciated.

In Fig. 12, the calculated waveform by the new model (10 iterations) is different from that by the full phase-domain model, especially at peaks. Because the errors of the new model listed in Tables 1 to 4 are very small, does the full phase-domain model show the inaccurate result?

Manuscript received February 25, 1998.

Bjørn Gustavsen and Adam Semlyen: We wish to thank Dr. Noda and Professor Ametani for their questions and remarks.

Their characterization of polar decomposition is correct: indeed, in a state equation formulation, the poles, i.e. the elements of matrix $A$ (together with the corresponding travel delays), are obtained from the eigenvalues of $H(s)$, while the input and output matrices $B$ and $C$ are obtained by $B C$-iterations. Thus polar decomposition is in fact a simplified way to obtain a nearly optimal state equation realization. It obviates the difficult and slow procedure of a full numerical optimization. If used as the initialization step for the latter, it will speed it up significantly.

The discussers point to the fact that the deviation between the simulated response in figure 12 by the polar decomposition model and the full phase-domain model is quite noticeable, despite the high accuracy suggested by tables 1 to 4 . Figure A shows the simulated voltages by the two approaches when increasing the order of the fitting for both methods. This made the deviation become somewhat smaller, as is seen in the figure. It should be noted that the deviation does not increase much with time. Figure B shows the simulated voltage by the two approaches in the time interval of $20-30 \mathrm{~ms}$.

Finally, we would like to add that all elements of $H$, and of $Y_{c}$, are fitted simultaneously, during the BC-iterations. Because a least squares approach is used, elements that are small in magnitude tend to become less accurately fitted, in a relative sense, than large elements.

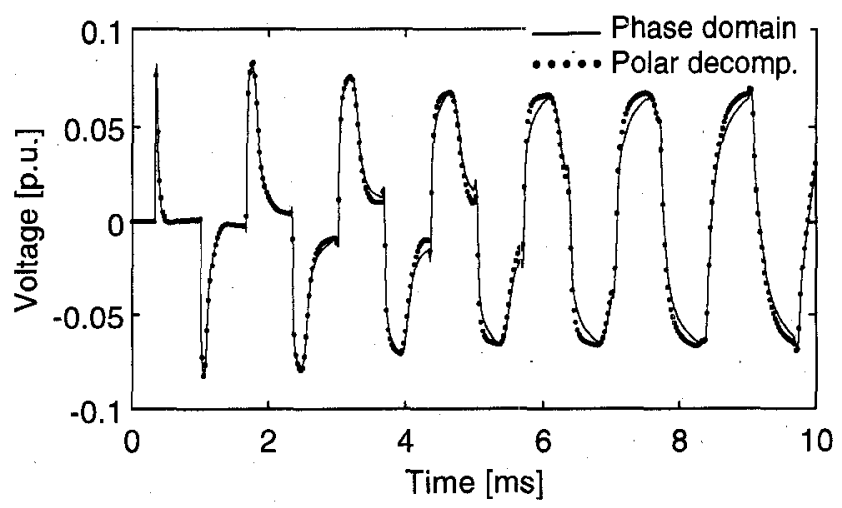

Fig. A Simulated voltage when increasing the order of the fiting

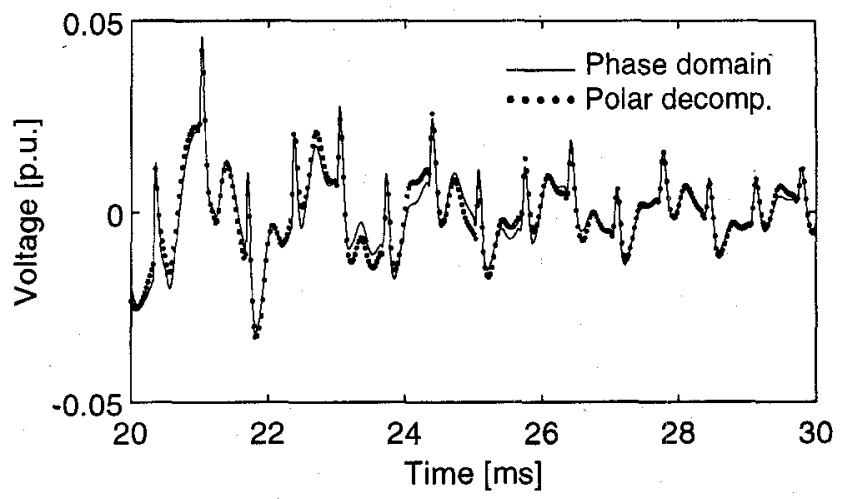

Fig. B Simulated voltage in the time interval $20-30 \mathrm{~ms}$ 